\title{
Total Serum Cholesterol and Pancreatic Cancer Risk: What Is the Link?
}

\author{
Raffaella Mormile ${ }^{1}$
}

Received: 18 May 2019 / Accepted: 23 June 2019 / Published online: 28 June 2019

(C) Arányi Lajos Foundation 2019

Total serum cholesterol (TSC) has recently been connected with pancreatic cancer risk [1]. It has been observed that the risk of developing pancreatic cancer increases when related to lower TSC independent of statins [1]. TSC has emerged to be a significant predictor of short-term risk for pancreatic cancer [1]. In this light, TSC has been proposed as a possible biomarker for risk stratification, screening, and early diagnosis of pancreatic cancer in future clinical prediction models [1]. TSC has been deemed a protective factor for overall survival in cancer patients [2]. However, the issue that an abnormal lipid profile is connected with cancer development is still under debate [2]. Pancreatic cancer represents the third leading cause of cancer death in the United States [1]. Inflammation represents a key player in tumor progression and metastasis in several tumor types including pancreatic cancer [3]. Interleukin-6 (IL-6) is a potent pro-inflammatory cytokine which appears to play a critical role in regulation of inflammation [3]. The development and progression of pancreatic cancer has been linked to IL-6 [3]. It has been found that IL-6 is expressed by many cellular sources within the pancreatic tumor microenvironment [3]. In pancreatic cancer, IL-6 is considered a strong activator of multiple aspects of tumor growth and progression [3]. It has been shown that IL-6 is elevated in the serum of patients with pancreatic cancer and relates to cachexia, advanced tumor stage and poor survival [4]. IL-6 has also been detected to promote pancreatic cancer cell migration [3, 4]. It has been demonstrated that IL-6 makes the tumor microenvironment permissive for metastatic dissemination [3, 4]. Several reports have highlighted that lipid levels, including total cholesterol levels, are altered under inflammatory conditions [5]. Inflammation has been proposed to reduce circulating lipid levels such as TSC via the induction of IL-6 [5]. Il-6 has been detected to influence lipid levels in the circulation [5]. IL-6 appears to exert different

Raffaella Mormile

raffaellamormile@alice.it

1 Division of Pediatrics and Neonatology, Moscati Hospital, Via A. Gramsci, 81031 Aversa, Italy effects on lipid metabolism in animals and humans [5]. IL-6 has been stated to reduce TSC [5]. On the contrary, it has been established that blockade of IL-6 increases blood levels of total cholesterol [5]. Taken together, I suppose that patients with higher TSC show the risk of pancreatic cancer reduced and independent of statins when compared with patients with lower levels of TSC as a result of decreased expression of IL-6. Thus, I hypothesize that a progressive reduction of TSC independent of diet and statins might indicate a recurrence even before new symptoms develop in patients affected by pancreatic cancer taking into account that pancreatic cancer typically develops with few symptoms in the early stage. Further research is warranted to better understand the potential interaction between TSC and IL-6 in the fight against pancreatic cancer.

\section{Compliance with Ethical Standards}

Conflict of Interest The author declares no potential conflicts of interest.

\section{References}

1. Chen WC, Boursi B, Mamtani R, Yang YX (2018) Total serum cholesterol and pancreatic cancer: a nested case-control study. Cancer Epidemiol Biomark Prev 28(2):363-369

2. Zhou P, Li B, Liu B, Chen T, Xiao J (2018) Prognostic role of serum total cholesterol and high-density lipoprotein cholesterol in cancer survivors: a systematic review and meta-analysis. Clin Chim Acta 477:94-104

3. Razidlo GL, Burton KM, McNiven MA (2018) Interleukin-6 promotes pancreatic cancer cell migration by rapidly activating the small GTPase CDC42. J Biol Chem 293(28):11143-11153

4. Holmer R, Goumas FA, Waetzig GH, Rose-John S, Kalthoff H (2014) Interleukin-6: a villain in the drama of pancreatic cancer development and progression. Hepatobiliary Pancreat Dis Int 13(4):371-380

5. Hashizume M, Mihara M (2011) IL-6 and lipid metabolism. Inflammation and Regeneration 31:325-333

Publisher's Note Springer Nature remains neutral with regard to jurisdictional claims in published maps and institutional affiliations. 